\title{
Patient considerations in the management of mental stress in psoriasis
}

This article was published in the following Dove Press journal:

Patient Intelligence

13 June 2012

Number of times this article has been viewed

\author{
Gerhard Schmid-Ott ${ }^{1,2}$ \\ Dana Böhm ${ }^{1,3}$ \\ Scott Stock Gissendanner ${ }^{1,2}$ \\ 'Hospital Management and Stress \\ Medicine, IREHA Institute for \\ Innovative Rehabilitation, Loehne, \\ Germany; '²Berolina Klinik, Loehne, \\ Germany; ${ }^{3}$ Saale Reha-Klinikum, \\ Bad Koesen, Germany
}

\begin{abstract}
Psoriasis is an incurable, inflammatory disease of the skin with a complex etiology. The disease has serious negative repercussions for patients' quality of life, but quality of life does not depend on physical symptoms alone. The impact of somatic symptoms on life quality is mitigated by various forms of mental stress. Through a discussion of the physical and mental burdens of psoriasis, the psychosocial determinants of life quality, common treatment options, and issues of adherence and resilience, this paper seeks to identify common sources of mental stress and appropriate strategies for stress management for psoriasis patients. The paper argues that a sustained and successful management of psoriasis depends on patients playing an active role in the prevention and reduction of their own personal sources of mental stress. Health-care professionals can and should assist patients in doing so.
\end{abstract}

Keywords: psoriasis, shared decision-making, treatment, adherence, resilience

\section{The burden of psoriasis}

Psoriasis is a chronic, inflammatory, recidivistic, and incurable disease of the skin that affects approximately $2 \%$ of the general population in Europe and North America. ${ }^{1}$ Characteristic outward symptoms of psoriasis vulgaris, the most common form, are erythema (reddening), desquamation (scaling), and induration (plaque) of the skin. Patients also experience uncomfortable sensations of itching and burning. Elbows, knees, lower back, navel, and scalp are most typically affected. Psoriasis is ultimately caused by as yet not well-understood multiple genetic and immunological factors, but its symptoms are triggered and exacerbated externally. Studies of twins estimate that genetic factors account for $60 \%-70 \%$ of an individual's risk of contraction. ${ }^{2}$

Psoriasis is considered a systemic ailment in the sense that those affected are also likely to suffer from one or more somatic and psychological comorbidities. ${ }^{1}$ Patients are more likely to be overweight or to have high blood pressure, high cholesterol levels, and diabetes. Young people with psoriasis have a higher long-term risk of heart disease than the same cohort in the general population, and psoriasis patients generally have a lower life expectancy than those with no skin ailment. Psychological or psychosomatic disorders, including, anxiety, depression, and Crohn's disease, are also more prevalent among those with psoriasis. There is also evidence of a higher prevalence of lifetime suicidal ideation among psoriasis patients due to disease-related suffering. ${ }^{3}$

Psoriasis affects all aspects of patients' well-being, not only due to the discomfort of its symptoms but also because its outwardly visible signs impair normal functioning in social interaction, recreation, employment, sexual relations, etc. Others' reactions
Correspondence: Scott Stock Gissendanner Berolina Klinik, Bültestrasse 2I,

32584 Löhne, Germany

Tel +49 573 I 782 I5 I

Email s.stockgissendanner@uglielje.de 
to these symptoms - even the mere anticipation of negative reactions - can have a profoundly negative emotional effect on patients, as consistently affirmed in empirical research. ${ }^{4-7}$ On the basis of survey instruments, psoriasis has been shown to have a negative effect on patients' quality of life that is at least as high as other dermatoses and equal to the impact of other diseases such as cancer. ${ }^{8-10}$ This high negative impact is traceable to a combination of symptom discomfort and psychosocial stress. Because somatic and mental aspects of the disease are closely connected, undesirable feedback effects (vicious circles) are possible whereby mental stress worsens somatic symptoms, which in turn increases mental stress, and so on.

The mental impact of psoriasis does not vary directly or necessarily with the objectively measurable symptoms of erythema, desquamation, and induration. ${ }^{5,11}$ Indeed, the negative effects of psoriasis play out on two distinct tracks: physical and mental. This means that two patients with a similar "objective" intensity of somatic severity, as measured by the same dermatologist, may perceive very different levels of life quality.

In sum, psoriasis is a lifelong disease that harms patients not only physically but also socially and emotionally. Indeed, from the patients' quality-of-life perspective, discussed below in greater detail, the most severe negative effects of psoriasis do not result from somatic symptoms alone, but rather from the interaction of physical symptoms and mental stress. If not managed well, these processes can lower quality of life and in some cases result in serious mental disorders. For psoriasis patients, quality of life thus depends in large measure on their ability to manage, together with their physicians, the psychological sequelae of the disease. Because the management and prevention of psychological dysfunction is at least as important as the management of objective dermatological symptoms, this paper reviews research into quality-of-life issues for psoriasis patients, treatment options and their psychological ramifications, and the role patients can play in the self-management of psychological symptoms over their entire life course.

\section{The focus of patient considerations: subjective quality of life}

Issues of patient considerations for the management of psoriasis are focused by a search for the correlates of patient perceptions of quality of life, as researchers seek a better understanding of why some patients are better able to shield themselves from the mental effects of persistent and recidivistic physical symptoms and lead more fulfilled lives. In the medical literature, "quality of life" is a multidimensional construct that includes physical, social, and emotional aspects of well-being. ${ }^{12}$ Issues of life quality and "soft" or subjective disease outcomes have been receiving greater attention in recent decades in scientific research, growing in importance relative to the more traditional, "hard" issues of etiology and somatic treatment. ${ }^{13}$

It is worth noting the different methods of measuring objective and subjective disease outcomes. The severity of somatic symptoms is measured clinically by trained professionals with diagnostic instruments such as the Physician Global Assessment or the very widely used Psoriasis Area and Severity Index. ${ }^{14}$ The latter measures the surface area affected and the degree of reddening, plaque, and scaling. ${ }^{15}$ Psoriasis patients' perceived quality of life is measured with patient survey instruments such as the Dermatology Life Quality Index or Skindex. The Dermatology Life Quality Index measures subjectively the degree to which a skin disease affected life quality during the previous week. It unites ten items into an overarching score, reflecting experiences in the subcategories of symptoms and feelings, daily activities, recreation, work and school, personal relationships, and treatment of the illness. ${ }^{16}$ Skindex is intended to measure the health-related quality of life of persons with skin maladies more comprehensively. Its original measures touched on the areas of cognitive effects, social effects, depression, fear, embarrassment, anger, physical discomfort, and physical limitations. ${ }^{17}$ These instruments ask patients to self-report their perceptions.

Even from the standpoint of etiology, but certainly also from the standpoint of quality of life, psoriasis is an individual-specific malady. Although its symptoms occur only among individuals who have a fixed, genetic predisposition to the disease, initial contraction and later flare-ups are triggered by a proximate, situation-specific "provocation" that can vary by physical location, personal health complications, occupational stress, and additional physical predispositions. Moreover, in addition to these kinds of provocations, which have their roots in primarily biological or environmental causes, other factors contributing to poor quality of life are rooted primarily in patients' subjective perceptions, especially of the experiences of stigmatization and other sources of psychosocial stress.

Research into the correlates of quality of life for psoriasis patients points consistently to four overlapping somatic and mental factors: clinically measurable somatic severity, subjectively perceived sensory skin symptoms, stigmatization, and stress. All of these are relevant to issues of patient 
self-management of the psychological burdens of psoriasis and are reviewed below.

The objectively measurable somatic symptoms of psoriasis can be worsened by many factors. Central among these are infections, medication, smoking, and high alcohol consumption. First of all, it has been shown that streptococcal and other kinds of infections often trigger the initial onset of psoriasis among children. Among adult psoriasis patients, infection also plays a role. Lesions can exacerbate themselves because they compromise locally the skin's natural infection barriers. Second, some patients experience worsening of symptoms after taking medicines. This effect has been reported for beta-blockers, angiotensin-converting-enzyme inhibitors, lithium, and chloroquin/hydroxychloroquin. ${ }^{1}$ Smoking, too, has been associated with the onset of psoriasis, possibly due to the immunological effects of nicotine. Cessation of smoking often brings a long-term improvement of symptom severity. This also means, however, that symptom improvement often begins only many months after quitting. Finally, alcohol is an issue for psoriasis patients. Consumption of alcohol in moderate amounts is unproblematic, but heavy consumption has been associated with more severe symptoms and less favorable treatment outcomes. ${ }^{18}$

The first of three psychological factors that impinge on quality of life is the perceived intensity of sensory symptoms. Sensory skin symptoms are determined in part by biological mechanisms, yet the perception of their intensity varies from patient to patient and can intensify or ebb over time for the same patient. Felt skin symptoms can be exacerbated by negative social experiences and emotions, and they play an important role in the determination of life quality. Sensory symptoms take the form of pruritus, skin pain, burning, and more generalized skin discomfort. Pruritus appears to be the worst of these symptoms and has a proven influence on well-being and psychological comorbidity. ${ }^{19}$ A secondary effect of sensory skin symptoms, sleep disruption, is also a major cause of concern for patients. Ljosaa and colleagues demonstrated that skin pain and skin discomfort are significantly related to quality of life but that this relationship is mediated through sleep disturbance. ${ }^{20}$ In other words, patients who wake at night due to discomfort and pain report a higher negative impact due to psoriasis than patients who can sleep through the night.

The second psychological factor, stigmatization, has been shown repeatedly to have a significant negative impact on psoriasis patients' quality of life..$^{21-23}$ A stigma is social rejection of a person on the basis of a distinguishing characteristic. For psoriasis patients, stigmatization can result from the reaction of others to the unsightliness of lesions and scaling or from the wrong belief that psoriasis is contagious. Patients are then shunned, subtly or overtly, and must deal with issues of "body-image, self-esteem and selfconcept, poor psychological adaptation, and feelings of ... shame and embarrassment concerning their appearance." ${ }^{12}$ Stigmatization can involve a number of mechanisms or dimensions. In Ginsburg and Link's widely used conceptualization, six are relevant: anticipation of rejection, feeling of being flawed, sensitivity to others' attitudes, guilt/shame, secretiveness, and positive attitudes. ${ }^{24}$

Psoriasis patients, even those with relatively mild symptoms, experience high stigmatization relative to sufferers of other skin maladies, with significant correlations with outcomes such as quality of life, depression, and disability. ${ }^{25,26}$ Patients who have lesions on parts of the body that are readily visible, especially the face and hands, are exposed to greater social stigmatization than patients whose lesions are easily hidden by normal clothing. Women and men are sensitive to different aspects of stigmatization, and women often report experiences of stigmatization more frequently than men. ${ }^{27,28}$ Past or continuing experiences with stigmatization puts pressure on patients to withdraw socially. ${ }^{21}$

The third mental factor thought to determine quality of life, stress, is equally serious. "Basically, anything that causes an individual to experience mental or physical stress can have an impact, including great joy or suffering, disease symptoms, problems at work, relationship problems, family troubles or social rejection." "Stress is always in some way both psychological and physical: even those forms of stress that have purely psychosocial causes ultimately have somatic implications, because they trigger immunological and endocrinological counterbalancing reactions in the body. Mental stress causes phenotypic changes in circulating lymphocytes and is regarded as an important trigger of psoriasis, known also as a Th1-polarized inflammatory skin disease. "Especially, a higher stress-induced increase of $\mathrm{CLA}^{+} \mathrm{T}$ and $\mathrm{CLA}^{+}$Natural Killer (NK) cells in the circulation of patients with psoriasis might point to an increased ability of T and NK cells in the presence of psoriasis to home in on the skin during mental stress." ${ }^{29}$ Studies have shown that the immunological reaction to physical stress among psoriasis patients is above normal, suggesting that psoriasis patients are especially sensitive to stress-inducing experiences. ${ }^{1}$ Stress may thus play a role both in provoking the initial onset of psoriasis as well as in influencing the severity of symptoms later on. ${ }^{30}$ Establishing empirically a direct causal connection between stress and the physical symptoms of psoriasis is difficult, 
however, "possibly because of considerable individual variation in coping skills and the importance of the emotional meaning rather than the intensity" of biographical events. ${ }^{18}$ Clinicians, therapists, and patients are advised to note the distinction between negative forms of stress ("distress") from healthy forms of stress caused, for example, by athletic activity or the feeling one gets while accomplishing challenging goals. Healthy stress is actually an important resource in the treatment of psoriasis, especially in the activation of personal mental resources.

In sum, it seems that while quality of life is tied to the severity of visible lesions, this impact is mediated by felt sensory perception, experiences with stigmatization, anticipation of stigmatization, and unhealthy personal coping strategies such as social withdrawal. The mediating impact of stigmatization is indeed well established in the literature, ${ }^{27,31}$ and this suggests a model (Figure 1) whereby objective physical symptoms exert effects on perceived quality of life both direct and mediated through sensory symptoms, stigmatization, and stress.

\section{Common therapies}

There is a great diversity in therapy regimens for psoriasis. Regimens include prevention through avoidance of provoking factors, topical treatments for affected skin areas, general external therapies (baths and light exposure), and systemic (internal) therapies. For patients with symptoms over a limited body area, moderate avoidance strategies and topical approaches are often sufficient. For more severe cases, the addition of systemic therapies is indicated. Psychosocial interventions are also common and appropriate for all levels of severity. Therapies and medications used in the treatment of psoriasis, which should be undertaken only under the supervision of a physician, are reviewed below so as to inform a discussion of their varying psychological implications.

First of all, patients do have a limited degree of control over their exposure to provoking factors, including the stress of social interaction. By avoiding provoking factors,

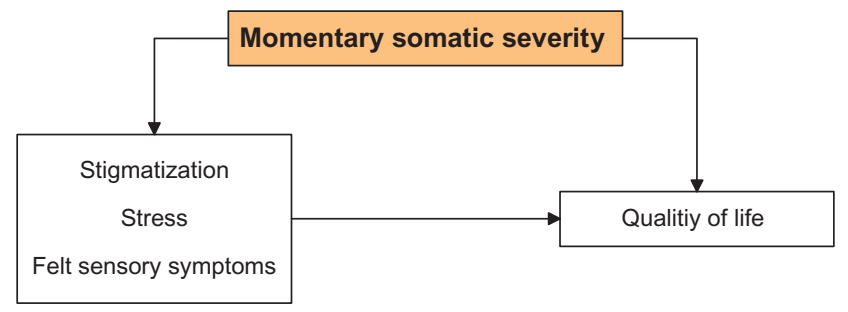

Figure I Model of direct and mediated effects of somatic severity on quality of life. symptom severity can in some cases be limited. But avoidance behaviors are not always healthy. When avoidance leads to persistent social withdrawal, seriously negative repercussions for mental health and life quality are possible.

For topical treatment of affected areas, the list of active ingredients with proven effectiveness encompasses four basic groups: corticosteroids, anthracene (coal tar) derivatives, vitamin $\mathrm{D}_{3}$ derivatives, and retinoids. Corticosteroids are related to the natural hormone cortisol, which has important functions in regulating the body's reaction to stress. It has a natural anti-inflammatory effect that is greatly enhanced in synthetic pharmaceuticals. An advantage of these drugs is that they can be mixed into a large number of preparations, including creams, lotions, and foams. Topically applied corticosteroids can quickly diminish psoriasis lesions and itching, and they are the most widely used type of drug in the treatment of psoriasis. ${ }^{1}$ Anthracene derivatives, including the medicine known internationally as dithranol, are chemically related to coal tar. Dithranol was in fact the first medicine developed for the treatment of psoriasis. It is used topically, often only in day clinics because of skin discoloration and risk of irritation. Stronger concentrations are used for shorter periods of time. Vitamin $\mathrm{D}_{3}$ regulates the body's calcium and phosphorous levels. It is important for metabolic processes involving bones and skin, and it is involved in regulating inflammation. ${ }^{1}$ In pharmaceutical preparations, its synthetic derivative calcipotriol is used in preparations for topical application. Retinoids are related to vitamin A and regulate cell growth and cell differentiation. Preparations with the active ingredient retinoic acid, used topically, are weaker in effect compared to the $\mathrm{D}_{3}$ derivatives and corticosteroids. Note also that not every patient responds to retinoid-based therapy.

In addition to these medicines, salicylic acid can be used to exfoliate psoriatic scales. It accelerates the shedding of epidermic cells and is thus used also for the treatment of other skin problems such as acne, dandruff, and calluses. The use of exfoliating preparations has two beneficial effects. ${ }^{1}$ First, by removing scales that cover lesion areas, the effectiveness of other therapies can be increased. Second, for some psoriasis patients, reducing unsightly scales is a major benefit in and of itself.

Going beyond specific treatments of lesion areas, patients may also make use of treatments that encompass the entire surface area of the skin. These have both healing and preventive effects. The most important general topical therapy for the skin is the regular use of lotions and creams that support the skin's natural protective functions. Patients who are 
undergoing therapies that can dry out or irritate the skin, such as light exposure, stand to benefit especially from daily skin care. Another general topical therapy, medicinal baths, is an ancient practice. Saltwater bathing especially can have a healing effect on psoriatic lesions, as saltwater seems to dissolve inflammatory chemicals present in the skin. ${ }^{1}$ Often, saltwater baths are combined with light therapies. The healing effect of light on skin lesions also has been appreciated for centuries, and today the use of lamps that emit ultraviolet light (UVA and UVB) belongs to the most widely used treatments of psoriasis. In psoralene + UVA therapy, patients are treated beforehand with psoralene creams or baths, which increase the skin's sensitivity to UVA light. In these treatments, caution is mandatory due to the carcinogenic properties of UVA radiation. Narrow-band "laser" light is also employed.

Systemic therapy, which employs internal medication, is appropriate only for more severe cases or when topical therapies are ineffective. ${ }^{1}$ Drugs approved for psoriasis treatment include retinoids, the immunosuppressant ciclosporin, acidic compounds known as fumarates, the antimetabolite and antifolate drug methotrexate, and new "biologics" engineered to emulate natural antibodies. These medications can be dangerous if improperly used and thus necessitate special care and regular monitoring by a physician.

\section{Psychological ramifications of different treatment regimens}

Treatment regimens that help patients manage both the physical symptoms and the psychological ramifications of psoriasis have long been recommended. ${ }^{32,33}$ Given the very individual nature of disease etiology and of patient responsiveness to the wide variety of available treatments, it is often a matter of months or years before a treatment can be found that patients consider optimal. Often, patients must settle for the best among several rather unattractive alternatives. Episodes during which trusted therapy regimens are no longer effective can occur, which in turn fosters frustration and resignation.

Corticosteroids bring the fastest, most effective relief of symptoms. However, since these applications should not be used on a long-term basis, the initial euphoria at the success of treatment is often followed by disappointment when treatment is terminated. Indeed, the rapid effect of the drugs may itself promote a form of psychological dependence in some patients. Dithranol, in contrast, has not yet been associated with negative side effects of long-term use. A major disadvantage of dithranol, however, is the brown staining of treated skin areas and clothing, which can increase patients' feelings of stigmatization and reduce adherence. Other topical preparations such as salicylic acid, vitamin $\mathrm{D}_{3}$ derivatives, and retinoids carry the advantage of quick application, but they can cause unpleasant sensations, such as burning. For some, the unpleasantness of these medications is worse than the disease's own symptoms. Systemic therapies, for their part, can have side effects ranging from the unpleasant to the extremely serious. Fumarates can disrupt the digestive tract. Methotrexate causes changes in the blood count, can damage liver and kidneys, terminates pregnancies, and disrupts ovulation and spermatogenesis. If a pregnancy occurs during retinoid therapy, congenital malformation is very likely. These and other potential side effects of systemic treatment can be mentally unsettling for patients looking for new treatment options, particularly for women of childbearing age, even when certain medications are strictly contraindicated and would never be prescribed. In contrast, light therapies (when properly dosed and supervised) and saltwater baths cause virtually no serious negative side effects and are much less distressing to patients. These therapies, however, are very time consuming and require a higher level of patience and perseverance.

For all these reasons, both the process of finding the right treatment for psoriasis and the treatment itself can cause patient distress. Physicians should take time to discuss in detail the advantages and disadvantages of different treatments with their patients with the goal of finding a treatment that not only minimizes the negative somatic side effects but that also avoids unnecessary psychological stress. A costbenefit analysis is a useful tool for this process. Experience has shown, additionally, that the best treatments are those that are supported and understood within the patient's social network. In the end, it is important to find a treatment or combination of treatments with which patients can identify personally in the sense that it gives them a feeling of control and personal efficacy in the treatment of the disease.

\section{Basic challenges in psoriasis management: adherence and resilience}

Psoriasis cannot be cured, but its somatic and psychological symptoms can be checked. With the goal of increasing perceived quality of life, patients can help themselves by finding, together with their doctor, the best personal medical treatment regimen and sticking to it. The main challenges to effective disease management can be expressed as adherence and resilience. Adherence is the extent to which patients follow the treatment regimen drawn up by their physician. Resilience refers to mechanisms for reducing the effects of psychosocial stressors that cannot be prevented or sensibly avoided. This involves finding the right personal balance between avoiding provoking factors and building 
self-confidence in daily social interaction. Although adherence and resilience are issues in the treatment of any disease, their psychosocial codeterminants are particularly important in the treatment of psoriasis.

Adherence reflects the positive circumstance that "a drug or therapy was given to the right person with the right explanation and the right information," but it can be hindered by a large number of factors, including undesired side effects, lack of effectiveness, nuisance of application, cost, mental distress, or simple forgetfulness. ${ }^{34}$ All of these negative factors are potential issues in the treatment of psoriasis, making adherence an especially great challenge for patients and doctors. Up to $50 \%$ of those with psoriasis do not take medications or observe treatment plans as directed; equally problematic is the possibility that many do not even begin treatment in the belief that betterment is medically impossible. ${ }^{35}$ Two main reasons for the stubbornness of adherence problems are the frustration patients feel with recurring symptoms and dissatisfaction with the medical treatment they receive. Krueger and colleagues found in a large sample of psoriasis patients that $40 \%$ were frustrated with the ineffectiveness of treatment. ${ }^{36}$ Many thought their treatment regimens were too mild. Yet even when treatments are effective for a while, gains made in reducing symptoms are often lost later, fueling frustration and defeatism and undermining patients' motivation to stay faithful to their treatment regimens.

Symptoms of mental stress common to psoriasis, such as depression and anxiety, are also negatively correlated with adherence. Mental stress is very persistent among psoriasis patients and is not necessarily improved even when treatments successfully reduce symptom severity. ${ }^{35}$ The centrality of psychosocial factors for adherence is also suggested by one finding that more severely affected patients are less likely to adhere to treatment regimens, ${ }^{37}$ arguably because these patients are more likely to feel powerless to manage the social ramifications of the disease. The persistence of mental stress is a particularly serious issue for adherence to those psoriasis treatments that must be maintained over long periods of time. Furthermore, since successful treatment also involves the avoidance of provoking factors, adherence requires a behavioral self-discipline that goes well beyond that required of individuals without skin ailments.

For psoriasis patients, maintaining adherence involves not only finding the most effective treatment but also openly discussing and coping with psychological stressors. For patients suffering from more severe psychological sequelae, professional psychiatric treatment that employs strategies such as psychotherapy, medication, or stress-reduction techniques is expected to improve adherence; indeed, for all patients, addressing the psychological aspects of care in an individual, personalized manner is recommended for improving adherence. ${ }^{38}$

Resilience is an interdisciplinary concept linking sociology, psychology, and medical research. Its domain encompasses all the personal resources that help individuals shield themselves from environmental stressors and ameliorate the effects of disease. Emotionally resilient individuals are those who "bounce back," or who experience emotional stress yet do not suffer from psychiatric complications such as depression, anxiety, or chronic fatigue as a result. They are able to recover their equilibrium after challenging events, and they have the capacity to "endure and continue forward in the face of adversity." ${ }^{39}$ But what makes healthier psoriasis patients more resilient? We know only that resilience is the product of a number of factors ranging from biochemical processes to family experiences to community support; research in the field is just now beginning to draw these processes together.

One study touching on the issue of resilience investigated the predictors of psoriasis patients' ability to construe benefits from negative events. The ability of patients to somehow find a deeper meaning or make sense of significant adversity is thought to help in making substantial and positive changes to worldviews and ultimately to promote healthy psychological adjustment. ${ }^{40}$ Positive effects in the study's sample of patients were linked to younger age, less alexithymia (deficiency in understanding and describing one's emotions), and being convinced that psoriasis is personally a chronic condition.

Other resilience-related studies have identified a possible connection between quality of life and the ability of patients to distance themselves emotionally from the socially isolating effects of psoriasis. Appropriate emotional distancing can be a source of resilience. Of particular interest is whether men are at an advantage in this respect. In a study comparing men's and women's expectations of discrimination based on their disease and their actual experiences with discrimination, Perrott and colleagues argue that women psoriasis patients suffer from a "double jeopardy." 41 They note that women are more likely than men to define themselves through their social connections to others, meaning that social rejection or the fear of social rejection sends out more severe emotional shock waves for women than for men. Women with psoriasis are indeed more likely to report feeling "upset" or "disturbed" in social settings. Women may well perceive 
stigmatization differently than men, meaning that either they are more sensitive to particular aspects of stigmatization or that they tend in fact to be stigmatized more frequently. ${ }^{27,28}$ Another source of difference lies in different perceptions among men and women of the effect of psoriasis on sexual relationships, whereby women are more likely to report sexual impairment due to the disease. ${ }^{18}$

\section{Can patient involvement in the management of psychological dysfunction enhance quality of life?}

Increasing attention is being paid to the positive effects of greater patient involvement in all aspects of care. Given the chronicity and complexity of psoriasis, patient involvement is especially relevant. Indeed, finding the right personal balance in treatment and prevention "requires a patient's full cooperation and active participation. ${ }^{35}$ Four aspects of patient orientation and patient involvement in psoriasis management are particularly promising for increasing quality of life: fitting treatment regimens to patients' personal lifestyles, improving patient-doctor interaction in the sense of shared decision-making (SDM), increasing availability of patient education programs, and activating self-help groups. For all these elements of patient involvement, their relevance in the management of psychological dysfunction is of central importance.

As noted above, the specific mix of advantages and disadvantages of psoriasis treatments is thought to play a central role in rates of adherence..$^{35}$ Importantly, the treatment itself can be a source of nuisance and stress for many patients, directly impairing quality of life. Patients appreciate treatments that are easy and short; they desire also to avoid side effects. However, there are no clear-cut guidelines regarding which particular kinds of treatments (eg, topical versus systemic) are more likely to limit mental stress. Rather, adherence seems to result when attractive treatment options (such as once-daily treatments) arise in combination with good psychological management leading also to a good understanding of the disease, faith in one's physician, acceptance of the disease, and acceptance of the limitations of treatment. ${ }^{35}$ One last factor, optimism about specific treatments, is associated with first-time use of treatment; this effect may work in favor of new drugs such as biologics. Yet patients and physicians should have a strategy for maintaining optimism even if a new treatment turns out to be disappointing.

Trust in one's physician and satisfaction with treatment increase adherence and are associated with higher quality of life. Treatment satisfaction seems closely related to the social skills of the physician generally, as patient satisfaction with treatment is higher among patients who give their doctors high marks for communication skills. ${ }^{35}$ This suggests that the quality of the working relationship between doctors and patients, including patient involvement in the sense of shared decision-making, is critical for understanding why some patients are better able than others to cope with psoriasis. The strategies and preferences of patients at the time when they initially seek help also matters; patients who feel isolated or stigmatized at the onset of symptoms interact with the health-care system and their physicians differently than patients who have greater social support. ${ }^{41}$

There is an ongoing discussion regarding SDM and its positive effects for adherence and treatment outcomes, and psoriasis is an especially good example of a disease that is best managed jointly. "The potential for side-effects and nuisance of treatment highlights the importance of joint planning and consensus between physician and patient in selecting treatments in line with lifestyle in order to optimize care ..."35 A recent German study demonstrated the wide variety of trade-offs involved in psoriasis treatment, concluding that patients may be willing to sacrifice effectiveness in order to maximize other aspects such as proximity of treatment facility. ${ }^{42}$ Some aspects of SDM are much more commonsensical but just as important. Some patients, for example, dislike lotions and creams; finding alternative delivery systems such as gels or switching to systemic treatments can increase treatment satisfaction and long-term adherence.

A third aspect of patient involvement involves educative programs designed to raise patients' understanding of disease causes, possible treatments, health-care system resources, and repercussions for mental and psychological states. Of all forms of psychosocial intervention intended to alleviate the emotional burden of psoriasis, patient education programs are widely considered to be economically the most effective. ${ }^{2,35,43}$ Improved patient education has been recommended for many years, and education programs are now standard adjunctive treatment in many places, including Germany. ${ }^{2,44}$ Psoriasis treatment combined with patient education has been shown to be more effective in improving clinical severity and reducing psychological comorbidities in comparison to treatment alone ${ }^{45}$ More information, clarification of values, and better decision-making skills have been cited by psoriasis patients as gaps that could be addressed through better education. ${ }^{46}$ Education programs that work also to improve basic health literacy and numeracy skills of patients are recommended, ${ }^{47}$ and these are especially relevant 
for understanding the potentially very wide range of dosage instructions for psoriasis medications taken by patients.

The fourth and final area in which patient involvement can be profitably improved is related to the work of self-help groups. There is to date little work quantifying the effect of participation in self-help groups or large psoriasis organizations, but a role in emotional support seems clear. ${ }^{18,48}$ They also provide important educative resources. An unanswered question is the extent to which participation in self-help groups fosters greater adherence or higher treatment satisfaction.

Richards and colleagues summarize these findings in a model of patient motivation and adherence (Figure 2). ${ }^{35}$ They hypothesize that adherence is enhanced by improved patient orientation in the areas of treatment selection, trust-building, and education. They suggest that simple treatments, patient education with written instructions, and good doctor-patient communication in the sense of collaborative management are the keys to higher rates of adherence, and ultimately also to higher quality of life. To this we can add the role of self-help groups in patient education and motivational support.

\section{Outlook}

We have seen that psoriasis is a lifelong disease that harms patients physically, socially, and emotionally. For patients, quality of life depends especially on the objective severity of

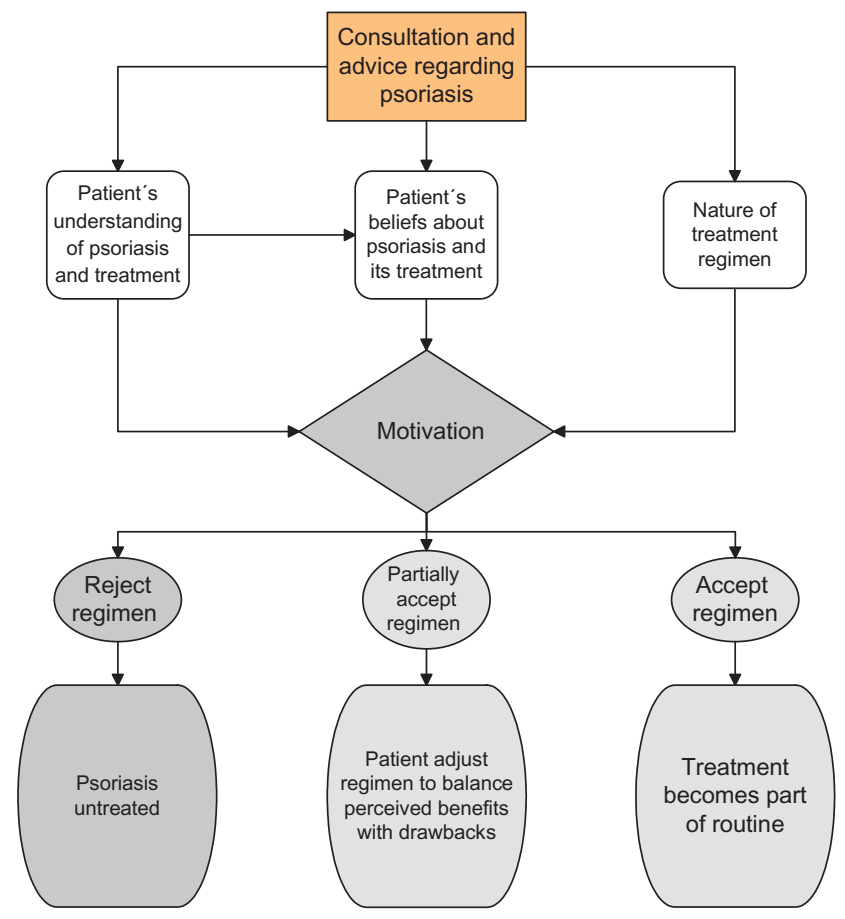

Figure 2 Model of patient motivation and adherence among psoriasis patients. Richards et al. 2006:376. ${ }^{35}$ (Published with permission from John Wiley and Sons). somatic symptoms, felt sensory symptoms, stigmatization, and stress. For addressing this complex set of factors, complex treatment regimens are necessary. Yet patients are often dissatisfied with treatment or discouraged by recurring symptoms, which reduces their adherence motivation. Also, some patients find it difficult to find a deeper personal meaning in their struggle with the disease and to distance themselves emotionally from the adverse impact of the disease on their social interaction.

What can be done, in light of this knowledge, to improve our ability to maximize patients' life quality and help them manage psychological dysfunction? A central challenge for future research is achieving a better understanding of the interaction of subjective and objective factors. This involves combining "hard science" investigation of endocrinological and immunological processes with approaches from sociology and psychology that help us understand the link between stigmatization, stress, and psychological sequelae. A main goal of further research will be finding ways to bolster economically adherence and resilience. A central aspect of this challenge is finding ways of getting patients involved in self-management economically and effectively with the goal of improving shared decision-making, increasing patient satisfaction with treatment regimens, and increasing patients' understanding of all aspects of the disease and its treatment. For all of these efforts, attention to the (self-)management of psychological stressors is of utmost importance.

Future research into psoriasis will be dominated by attempts to better understand the complex etiology of the disease and how to seize the benefits of better patient incorporation in all aspects of its management. It is to be hoped that increasingly better models will emerge, moving us toward a "grand theory" that better unites our currently segmented knowledge of genetic, molecular, environmental, and psychosocial processes.

\section{Disclosure}

The authors report no conflicts of interest in this work.

\section{References}

1. Mrowietz U, Schmid-Ott G. Schuppenflechte. Was Sie schon immer über Psoriasis wissen wollten (What You Always Wanted to Know About Psoriasis). 3rd ed. Basel: Karger; 2011. (German)

2. Nast A, Boehncke W, Mrowietz U, et al. S3-guidelines for the treatment of psoriasis vulgaris. Update 2011 German. J Dtsch Dermatol Ges. 2011; 9 Suppl 2:S1-S104.

3. Rapp SR, Exum ML, Reboussin DM, et al. The physical, psychological and social impact of psoriasis. J Health Psychol. 1997;2:525-537. 
4. Fortune DG, Richards HL, Griffiths CE. Psychologic factors in psoriasis: consequences, mechanisms, and interventions. Dermatol Clin. 2005;23:681-694.

5. Kimball AB, Jacobson C, Weiss $\mathrm{S}$, et al. The psychosocial burden of psoriasis. Am J Clin Dermatol. 2005;6:383-392.

6. Magin P, Adams J, Heading G, et al. The psychological sequelae of psoriasis: results of a qualitative study. Psychol Health Med. 2009; $14: 150-161$

7. Schmid-Ott G, Malewski P, Kreiselmaier I, Mrowietz U. Psychosocial consequences of psoriasis - an empirical study of disease burden in 3753 affected people. Hautarzt. 2005;56:466-472. German.

8. Misery L, Thomas L, Jullien D, et al. Comparative study of stress and quality of life in outpatients consulting for different dermatoses in 5 academic departments of dermatology. Eur J Dermatol. 2008;18: 412-415.

9. Rapp SR, Feldman SR, Exum ML, et al. Psoriasis causes as much disability as other major medical diseases. J Am Acad Dermatol. 1999;41:401-407.

10. Weiss SC, Kimball AB, Liewehr DJ, et al. Quantifying the harmful effect of psoriasis on health-related quality of life. JAm Acad Dermatol. 2002;47:512-518.

11. Reimus JL, Vingerhoets AJ, Soons PH, Korstanje MJ. Suffering in psoriasis patients: its relation with illness severity and subjective well-being. Int J Dermatol. 2007;46:1042-1045.

12. Ashcroft DM, Li Wan PA, Williams HC, Griffiths CE. Quality of life measures in psoriasis: a critical appraisal of their quality. J Clin Pharm Ther. 1998;23:391-398.

13. McKenna KE, Stern RS. The outcomes movement and new measures of the severity of psoriasis. J Am Acad Dermatol. 1996;34:534-538.

14. Gottlieb AB, Chaudhari U, Baker DG, et al. The National Psoriasis Foundation Psoriasis Score (NPF-PS) system versus the Psoriasis Area Severity Index (PASI) and Physician's Global Assessment (PGA): a comparison. J Drugs Dermatol. 2003;2:260-266.

15. Schmitt J, Wozel G. The psoriasis area and severity index is the adequate criterion to define severity in chronic plaque-type psoriasis. Dermatology. 2005;210:194-199.

16. Finlay AY, Khan GK. Dermatology Life Quality Index (DLQI) - a simple practical measure for routine clinical use. Clin Exp Dermatol. 1994;19:210-216.

17. Chren MM, Lasek RJ, Quinn LM, et al. Skindex, a quality-of-life measure for patients with skin disease: reliability, validity, and responsiveness. J Invest Dermatol. 1996;107:707-713.

18. Basavaraj KH, Navya MA, Rashmi R. Stress and quality of life in psoriasis: an update. Int J Dermatol. 2011;50:783-792.

19. Reich A, Hrehorow E, Szepietowski JC. Pruritus is an important factor negatively influencing the well-being of psoriatic patients. Acta Derm Venereol. 2010;90:257-263.

20. Ljosaa TM, Mork C, Stubhaug A, et al. Skin pain and skin discomfort is associated with quality of life in patients with psoriasis. $J$ Eur Acad Dermatol Venereol. 2012;26:29-35.

21. Hrehorow E, Salomon J, Matusiak L, et al. Patients with psoriasis feel stigmatized. Acta Derm Venereol. 2011;92:67-72.

22. Perrott SB, Murray AH, Lowe J, Mathieson CM. The psychosocial impact of psoriasis: physical severity, quality of life, and stigmatization Physiol Behav. 2000;70:567-571.

23. Schmid-Ott G, Burchard R, Niederauer HH, et al. Stigmatisierungsgefühl und Lebensqualität bei Patienten mit Psoriasis und Neurodermitis. (Stigmatization and quality of life of patients with psoriasis and atopic dermatitis). Hautarzt. 2003;54:852-857. (German)

24. Ginsburg IH, Link BG. Feelings of stigmatization in patients with psoriasis. J Am Acad Dermatol. 1989;20:53-63.

25. Gupta MA, Gupta AK, Watteel GN. Perceived deprivation of social touch in psoriasis is associated with greater psychologic morbidity: an index of the stigma experience in dermatologic disorders. Cutis. 1998;61:339-342.
26. Richards HL, Fortune DG, Griffiths CE, Main CJ. The contribution of perceptions of stigmatisation to disability in patients with psoriasis. J Psychosom Res. 2001;50:11-15.

27. Böhm D, Stock Gissendanner S, Snitjer K, et al. Perceived relationships between severity of psoriasis symptoms, gender, stigmatization and quality of life. J Eur Acad Dermatol Venereol. Epub February 14, 2012.

28. Schmid-Ott G, Kunsebeck HW, Jager B, et al. Significance of the stigmatization experience of psoriasis patients: a 1-year follow-up of the illness and its psychosocial consequences in men and women. Acta Derm Venereol. 2005;85:27-32.

29. Schmid-Ott G, Jaeger B, Boehm T, et al. Immunological effects of stress in psoriasis. Br J Dermatol. 2009;160:782-785.

30. Zachariae R, Zachariae H, Blomqvist K, et al. Self-reported stress reactivity and psoriasis-related stress of Nordic psoriasis sufferers. J Eur Acad Dermatol Venereol. 2004;18:27-36.

31. Vardy D, Besser A, Amir M, et al. Experiences of stigmatization play a role in mediating the impact of disease severity on quality of life in psoriasis patients. Br J Dermatol. 2002;147:736-742.

32. Farber EM, Nall L. Psoriasis: a stress-related disease. Cutis. 1993;51: 322-326.

33. Russo PA, Ilchef R, Cooper AJ. Psychiatric morbidity in psoriasis: a review. Australas J Dermatol. 2004;45:155-159.

34. van Dongen N. Let's be effective, let the patients talk! Does 'patient intelligence' have an effect on improvements in quality within the healthcare environment? Patient Intell. 2009;1:1-5.

35. Richards HL, Fortune DG, Griffiths CE. Adherence to treatment in patients with psoriasis. J Eur Acad Dermatol Venereol. 2006;20: 370-379.

36. Krueger G, Koo J, Lebwohl M, et al. The impact of psoriasis on quality of life: results of a 1998 National Psoriasis Foundation patientmembership survey. Arch Dermatol. 2001;137:280-284.

37. Zaghloul SS, Goodfield MJ. Objective assessment of compliance with psoriasis treatment. Arch Dermatol. 2004;140:408-414.

38. Bewley A, Page B. Maximizing patient adherence for optimal outcomes in psoriasis. J Eur Acad Dermatol Venereol. 2011;25 Suppl 4:9-14.

39. Zautra AJ. Resilience: one part recovery, two parts sustainability. J Pers. 2009;77:1935-1943

40. Fortune DG, Richards HL, Griffiths CE, Main CJ. Adversarial growth in patients undergoing treatment for psoriasis: a prospective study of the ability of patients to construe benefits from negative events. Psychol Health Med. 2005;10:44-56.

41. Schmid-Ott G, Jager B, Kunsebeck HW, et al. Illness experience and treatment seeking behavior of psoriasis patients. Hautarzt. 1997; 48:547-555. German.

42. Schaarschmidt ML, Schmieder A, Umar N, et al. Patient preferences for psoriasis treatments: process characteristics can outweigh outcome attributes. Arch Dermatol. 2011;147:1285-1294.

43. Schmid-Ott G, Keins P, Fartasch M, et al. Atopic eczema prevention program in childhood and adolescence - a model project of the German government. Dermatol Psychosom. 2000;1:179-182.

44. Farber EM, Nall L. The office visit and the self-help concept in treating the patient with psoriasis: a strategy revisited. Cutis. 1993;51: 236-240.

45. Fortune DG, Richards HL, Kirby B, et al. A cognitive-behavioural symptom management programme as an adjunct in psoriasis therapy. Br J Dermatol. 2002;146:458-465.

46. Tan J, Stacey D, Fung K, et al. Treatment decision needs of psoriasis patients: cross-sectional survey. J Cutan Med Surg. 2010;14: 233-239.

47. Bosworth $\mathrm{H}$. Challenges and strategies to improve patient health literacy and competencies. Patient Intell. 2010;2:19-25.

48. Schmid-Ott G, Schallmayer S, Calliess IT. Quality of life in patients with psoriasis and psoriasis arthritis with a special focus on stigmatization experience. Clin Dermatol. 2007;25:547-554. 


\section{Publish your work in this journal}

Patient Intelligence is an international, peer-reviewed, open access journal that characterizes and measures the central role of patient behavior and intention in optimizing healthcare management in all areas of disease and complaint types. An improved understanding of patient intelligence coupled with predictive analysis helps an organization contribute more effectively to achieving better outcomes.

The journal is characterized by the rapid reporting of reviews, original research, methodologies, analytics, modeling, clinical studies and patient surveys across all disease areas. The manuscript management system is completely online and includes a very quick and fair peer-review system. Visit http://www.dovepress.com/ testimonials.php to read real quotes from published authors.

Submit your manuscript here: http://www.dovepress.com/patient-intelligence-journal 